\title{
A study on Healthy Breathing Pattern when wearing a mask
}

\author{
Zhixing Tian', Bong-Young Kim² and Myung-Jin Bae ${ }^{3 *}$ \\ ${ }^{1}$ Soong-sil University, Department of Information and telecommunication Engineering, Seoul, 06978, Korea. \\ Orcid Id : 0000-0003-3882-2459 \\ ${ }^{2}$ Soong-sil University, Department of Information and telecommunication Engineering, Seoul, 06978, Korea. \\ Orcid Id : 0000-0002-3553-039X \\ ${ }^{3}$ Soong-sil University, Department of Information and telecommunication Engineering, Seoul, 06978, Korea. \\ Orcid Id : 0000-0002-7585-0400
}

\begin{abstract}
:
After the COVID-19 pandemic, wearing masks is still an important means of epidemic prevention. It is advocated by the World Health Organization and many countries. For a long time, people's lives are inseparable from the masks, and may still last for a long time. Should not overemphasize its protective role and ignore its problems. Many problems caused by masks are mostly related to breathing. Masks change people's breathing environment, and a more closed breathing space increases breathing resistance. Therefore, it is necessary to change the autonomous breathing pattern in response to changes in the respiratory environment. The new breathing pattern was proposed by Sori Sound Engineering Lab. The new active breathing method, combined with the advantages of deep breathing to relieve pressure, extends the inhalation and exhalation time, allows the air in the mask to exchange with the outside air for sufficient time, improves the air exchange rate, and reduces the air resistance. It can effectively reduce the health effects of wearing a mask. Relieve the pressure induced by wearing a mask. Blood oxygen saturation measurement and HRV measurement prove that the new breathing pattern is indeed effective.
\end{abstract}

Keyword: COVID-19, Mask, Stress, Active Control of Breathing Pattern, Blood Oxygen Saturation, HRV

\section{INTRODUCTION}

It has been several months since the COVID-19 pandemic, and the epidemic situation in many countries has been effectively controlled, but there is still the possibility of another outbreak, so governments recommend that people wear masks. Wearing masks in public has become a regulation and has become part of people's daily lives. For a long time, the problem of wearing masks has also received more attention. For example, wearing a mask for a long time can cause problems such as poor breathing, respiratory infections, and skin allergies. The problem of pressure induced by wearing a mask is becoming more and more serious, which causes many people to resist using it. This is not good for epidemic prevention. The high probability is caused by increased respiratory resistance and mild hypoxia. Because the use of masks can hinder the exchange of oxygen and carbon dioxide in the lungs, for example, studies have shown that after wearing N95, the resistance to inspiratory and expiratory flow increases by an average of $126 \%$ and $122 \%$, respectively. The air exchange volume was reduced by an average of $37 \%$ [1]. Therefore, the breathing method when wearing a mask is particularly important. When people wear a mask, the breathing resistance increases. If people still use the standard autonomous breathing pattern unchanged, it is easy to feel respiratory discomfort or slight hypoxia. However, active control of breathing can improve this situation very well. The Sound Lab has proposed a breathing pattern that allows people to breathe healthily when wearing a mask. It allows people to wear masks to increase the exchange rate of air, obtain more oxygen, and reduce masks effects on obstruction of breathing. Of course, this also effectively prevents the pressure induced by wearing a mask. This method is simple and applicable without additional cost. It is suitable for people who wear masks for a long time but can not take off the masks at any time to breathe fresh air. This active breathing pattern can bring them relaxed feelings [2].

This paper aims to propose a healthy breathing pattern when wearing a mask, which can help more people who are troubled by wearing a mask. The reasons for the pressure induced by the mask are explained from the physiological and psychological aspects, and the process of how to solve these problems is explained by the new breathing pattern. Using blood oxygen saturation measurement and HRV measurement to reflect the new breathing pattern can increase the amount of oxygen inhaled and relieve pressure. Finally, summarize the full text.

\section{CAUSES OF PRESSURE INDUCED BY WEARING A MASK}

\subsection{PSYCHOLOGICAL REASONS}

The N95 mask that people often wear is a breathing filter device that can filter particles with aerodynamic diameter and physical diameter of $0.075 \mu \mathrm{m} \pm 0.020 \mu \mathrm{m}$ to reach more than $95 \%$. It has 
good protection against PM2.5 and viruses, but it has good airtightness and has a high hindrance to breathing. After wearing the N95 mask, the inspiratory and expiratory flow resistance increased by an average of $126 \%$ and $122 \%$, respectively. Higher exhalation and inhalation resistance will reduce the comfort of breathing. Lower air volume will mean that the wearer may need to inhale harder to obtain the required amount of fresh air. This can be explained by the formula of airway resistance. From the formula, the airway resistance is proportional to the pressure difference and inversely proportional to the flow velocity and cross-sectional area. Masks block the exchange of lung air with external air, which is equivalent to reducing the cross-sectional area (s) of the external breathing channel and reducing the air velocity (v), so the respiratory resistance increases, which can cause discomfort and induce psychological pressure [3] [4]

$$
\begin{aligned}
& R_{A W}=\frac{\Delta P}{\dot{V}} \\
& \text { Where } \Delta P=P_{A T M}-P_{A}, \text { and } \dot{V}=v * S \\
& \text { Thus, } R_{A W}=\frac{P_{A T M}-P_{A}}{v * S}
\end{aligned}
$$

$R_{A W}$ is Airway Resistance, $P_{A T M}$ is Atmospheric Pressure, $P_{A}$ is Alveolar Pressure. $v$ is flow velocity, and $s$ is cross-

sectional vector area.

\subsection{PHYSIOLOGICAL REASONS}

After wearing the N95 mask, the air exchange volume was reduced by an average of $37 \%$. This is due to the geometric dead space in the mask. Because the mask hinders the diffusion of gas, a portion of the carbon dioxide will remain in the geometric dead space and cannot be discharged, further causing this part of carbon dioxide to be inhaled when inhaling again. The concentration of carbon dioxide in the inhaled air is higher than before, and the amount of oxygen obtained by the body per unit time is reduced, which may cause mild hypoxia. Especially when a large amount of mental activities and sports consume a lot of oxygen, it may be due to hypoxia Cause headaches, dizziness, nausea, and increase the physiological and psychological pressure of the body [5] [6].

\section{NEW ACTIVE CONTROL BREATHING PATTERN}

The normal breathing pattern is to use the nose to breathe, and the breathing frequency is 15 to 16 times per minute. The new breathing pattern is to inhale with the nose and exhale with the mouth. The breathing rate is 6-8 times per minute, inhale deeply for 4 seconds, and hold the breath for 2 seconds. Sound for 7 seconds. The new breathing pattern is similar to the normal deep breathing method, but requires a vibrating vocal cord to sound when exhaling. Controlling vocal cord vocalization restricts the outflow of airflow, so that the exhalation time is longer than that of ordinary deep breathing, and the breathing time is 2 to 3 seconds longer than ordinary deep breathing. Too much carbon dioxide can have more time to diffuse from the mask to the outside world, reducing the amount of carbon dioxide inhaled during the next inhalation. At the same time, deep inhalation increases the pressure of the lung muscles, the pressure in the lung ( $\left.\mathrm{P}_{-} \mathrm{A}\right)$ increases, and the pressure difference between the lung and the outside world becomes smaller. And deep breathing can speed up the air flow rate (v), and airway resistance (R_AW) decreases, then oxygen inhalation will become easier, and the increase in inhalation time will also increase the amount of inhaled oxygen. The reduction in airway resistance makes breathing easier, and longer breathing time also increases the amount of air exchanged, and people can get more carbon dioxide and oxygen inhalation. It is recommended to take an active controlled breathing pattern every hour, every 5 minutes [7].

\section{BLOOD OXYGEN SATURATION MEASUREMENT}

Using photoelectric oximeter, we measured the blood oxygen saturation of normal breathing without mask, normal breathing with mask and wear a mask to actively control breathing. The subjects were healthy persons aged from 20 to 30 years old, 5 men and 5 women. The test duration in each case is 5 minutes

\begin{tabular}{|c|c|c|c|}
\hline & \multirow{2}{*}{$\begin{array}{c}\text { No mask } \\
\text { Spontaneous } \\
\text { breathing } \\
\text { pattern } \\
\mathrm{SPO}_{2}(\%)\end{array}$} & \multicolumn{2}{|c|}{ Keep mask on } \\
\hline & & $\begin{array}{c}\text { Spontaneous } \\
\text { breathing } \\
\text { pattern } \\
\mathrm{SPO}_{2}(\%)\end{array}$ & $\begin{array}{c}\text { Control } \\
\text { breathing } \\
\text { pattern } \\
\mathrm{SPO}_{2}(\%)\end{array}$ \\
\hline 1 & 98 & 96 & 98 \\
\hline 2 & 99 & 97 & 99 \\
\hline 3 & 98 & 97 & 98 \\
\hline 4 & 98 & 96 & 98 \\
\hline 5 & 97 & 95 & 96 \\
\hline 6 & 98 & 96 & 98 \\
\hline 7 & 99 & 97 & 99 \\
\hline 8 & 99 & 98 & 99 \\
\hline 9 & 98 & 96 & 99 \\
\hline 10 & 97 & 95 & 97 \\
\hline
\end{tabular}
[8]. Table 1 shows the blood oxygen test results.

Table 1. Blood oxygen test results 
The standard arterial oxygen saturation is $98 \%$. And when it less than $94 \%$ is hypoxic [6]. It can be seen from the table that when not wearing, most of the testees are standard values. When wearing a mask, the blood oxygen saturation drops, but they are all above $94 \%$, which shows that wearing a mask does hinder oxygen intake. Due to the regulation of spontaneous breathing, low oxygen consumption in quiet sitting did not cause severe hypoxia. However, when the testee begins to control breathing, the new breathing pattern can restore the blood oxygen saturation to the standard value. In other words, the new breathing pattern improves the efficiency of gas exchange and increases the amount of oxygen absorbed when wearing a mask.

\section{HRV TEST AND RESULT}

\subsection{DEFINITION OF HRV}

The autonomic nervous system is composed of sympathetic nerves and parasympathetic nerves. The two have opposite effects. The sympathetic nerves have a promoting effect, which keeps the body alert, improves concentration, and achieves a state of active response. On the contrary, the parasympathetic nerve has an inhibitory effect, which is responsible for letting people relax, save energy, promote digestion, and initiate sleep. Studies have shown that when a person has a stress response, the stressor will reduce parasympathetic nerve activity. people will feel alert, nervous, and tired because they cannot relax. Heart rate variability (HRV) refers to the degree of fluctuation of the interval between heartbeats. That is, the length of the NN interval changes. Figure 1 is the NN interval. HRV can detect parasympathetic nerve activity, and the stressors will reduce parasympathetic nerve activity, which translates into a lower HRV. Conversely, when people relax, they show a higher HRV. Therefore, HRV can well reflect people's stress status [9] [10].

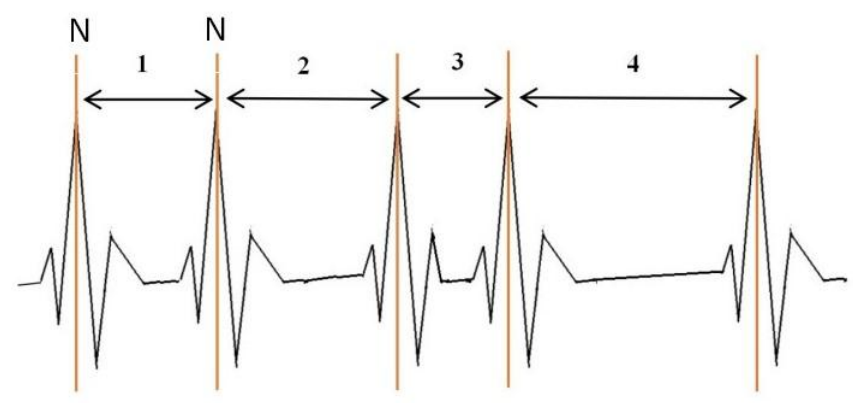

Figure 1. Interbeat Interval Series

There are many ways to quantify HRV. SDNN is a type of HRV time domain analysis that calculates the standard deviation of the NN interval in the time domain. The standard deviation reflects the degree of deviation from the average, so it can well reflect the degree of change of the NN interval. The following is the formula for calculating SDNN [11]. It is only necessary to measure the heart rate to calculate the SDNN.

$$
\begin{gathered}
\mathrm{N}-\mathrm{N} \text { Interval }(\mathrm{ms})=\frac{60000}{\text { Heart Rate }(\text { bpm })} \\
\operatorname{SDNN}(\sigma)=\sqrt{\frac{\sum_{k=1}^{N}\left(x_{k}-\mu\right)^{2}}{N}}
\end{gathered}
$$

where $x_{k}$ is k's N-N Interval, and $\mu$ is N-N Interval's Mean, $\mathrm{N}$ is total number of N-N Interval

\subsection{SDNN TEST RESULTS}

Experiment equipment are N95 mask and Pulse Oximeter. The heart rate and heartbeat interval can be extracted from the blood oxygen saturation signal, and then the collected heart rate data can be calculated in Matlab using the SDNN calculation formula [12]. The measurement data format is shown in Figure 2.

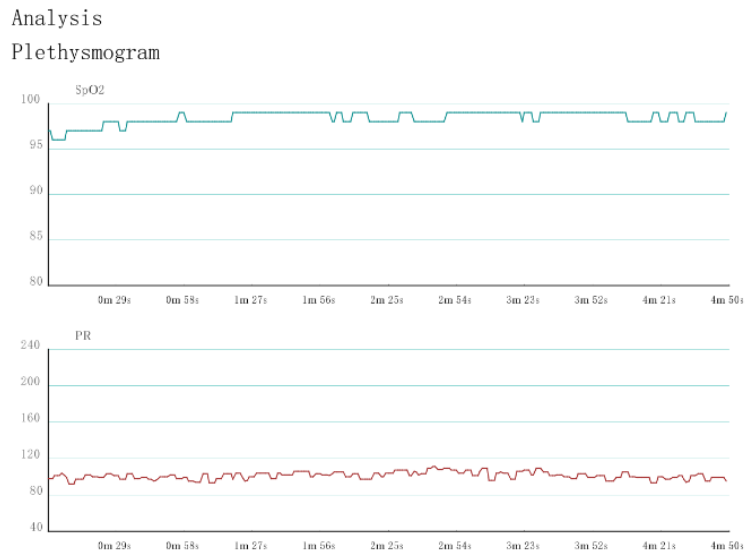

Figure 2. Measured blood oxygen saturation(green) and heart rate(red)

The testees were 10 healthy men and women aged 20 to 30,5 men and 5 women. The test environment is in a quiet laboratory, and all testees sit quietly. The test is divided into three parts. Part 1: Measure to breathe normally for 5 minutes while wearing a mask. Part 2: Use the mask to use the new breathing pattern for 5 minutes. Part 3: After two minutes of rest, measure the normal breathing again with a mask for 5 minutes. Because the breathing pattern affects the measured value of HRV, the second part is not measured. Finally compare the HRV value that before and after using the new breathing method [13]. Table 1 is the test results of HRV. 
Table 2. Results of Heart Rate measurement

\begin{tabular}{|c|c|c|c|c|c|}
\hline \multirow[b]{2}{*}{ Testees } & \multicolumn{2}{|c|}{ Before New Breathing Pattern } & \multicolumn{2}{|c|}{ After New Breathing Pattern } & \multirow[b]{2}{*}{$\begin{array}{l}\triangle S D N N \\
=\text { S2-S1 }\end{array}$} \\
\hline & $\begin{array}{l}\text { Average heart } \\
\text { rate(bpm) }\end{array}$ & SDNN 1(S1) & $\begin{array}{l}\text { Average heart } \\
\text { rate(bpm) }\end{array}$ & SDNN 2(S2) & \\
\hline 1 & 96 & 27.86 & 90 & 31.51 & 3.65 \\
\hline 2 & 87 & 23.41 & 84 & 30.87 & 7.46 \\
\hline 3 & 89 & 15.02 & 85 & 23.05 & 8.03 \\
\hline 4 & 93 & 13.82 & 83 & 21.71 & 7.89 \\
\hline 5 & 95 & 24.59 & 90 & 25.62 & 1.03 \\
\hline 6 & 84 & 35.14 & 82 & 48.13 & 12.99 \\
\hline 7 & 92 & 40.54 & 85 & 46.15 & 5.61 \\
\hline 8 & 99 & 38.41 & 92 & 47.57 & 9.16 \\
\hline 9 & 78 & 45.73 & 73 & 43.28 & -2.45 \\
\hline 10 & 66 & 39.04 & 64 & 54.53 & 15.49 \\
\hline
\end{tabular}

According to the data in the table, after using the new breathing pattern, the heart rhythm of all the testees is reduced. 9 people saw an increase in the SDNN value, and the number of SDNN rises accounted for $90 \%$ of the total number of people tested. The average rising SDNN for 9 people is 7.9. The experiment found that the new breathing pattern can increase HRV, which also shows that the new breathing pattern can really reduce the pressure. The new breathing pattern is a healthy breathing method when wearing a mask.

\section{CONCLUSION}

In the recent epidemic period, it seems that the need to continue wearing masks for several months and more is inevitable. The role of masks in preventing the spread of the epidemic has been sufficiently emphasized, but the problems caused by wearing masks for a long time have not caused too much attention, the new breathing pattern proposed by this paper is aimed at improving these problems. Designed to reduce the side effects of masks and protect people's health. This paper explains the mechanisms of masks, affecting breathing, and inducing stress. Many studies believe that in daily use, the psychological pressure caused by masks is greater than physiological pressure. The paper also proves this by detecting changes in blood oxygen saturation when sitting quietly. For the monitoring of psychological pressure, the HRV measurement method was used. After the experimental results used the new breathing pattern, HRV increased. The new breathing pattern relieves the pressure induced by wearing a mask. We recommend a new breathing pattern of 5 minutes every hour. This is beneficial to people's health [14] [15].

With the arrival of summer, the air in the mask will become more sultry and humid, which will also increase people's psychological pressure. In future research, we will continue to discuss the influence of air temperature and humidity on the psychological pressure.

\section{REFERENCE}

[1] Heow Pueh Lee, De Yun Wang, "Objective Assessment of Increase in Breathing Resistance of N95 Respirators on Human Subjects", The Annals of Occupational Hygiene, Volume 55, Issue 8, October 2011, Pages 917-921.

[2] Ravinder Jerath *, John W. Edry, Vernon A. Barnes, Vandna Jerath. "Physiology of long pranayamic breathing: Neural respiratory elements may provide a mechanism that explains how slow deep breathing shifts the autonomic nervous system ". Medical Hypotheses Volume 67, Issue 3, 2006, Pages 566-571.

[3] Kirkby, J.; et al. (2010). "Reference equations for specific airway resistance in children: the Asthma UK initiative". European Respiratory Journal. 36 (3): 622-629.

[4] Zhixing Tian, Bong-Young Kim, Myung-Jin Bae. "A Study on the Effect of Wearing Masks on Stress Response". International Journal of Engineering Research and Technology 13(4), pp. 807-813.

[5] Zhixing Tian, Bong-Young Kim, Myung-Jin Bae. "Study on Reducing the Stress of Wearing a Mask through Deep Breathing". International Journal of Engineering Research and Technology 13(4), pp. 756-761.

[6] By Jane E. Brody "Looking at Masks and Respiratory Health". The New Nork Times. [https://www.nytimes.com/2020/06/15/well/live/breathin g-masks-coronavirus.html?auth=link-dismissgoogle1tap]

[7] Bong-Young Kim, Ik-Soo Ahn and Myung-Jin Bae. " A Study on Lung Function Activation of Sound Necklace". Journal of Engineering and Applied Sciences (2018) Volume: 13, Issue: 3 .

[8] "Breathe better to move better: Train to breathe like a pro athlete" By Dana Santas, Special to CNN. [https://edition.cnn.com/2015/10/08/health/breathe-likepro-athlete/index.html] 
International Journal of Engineering Research and Technology. ISSN 0974-3154, Volume 13, Number 7 (2020), pp. $1562-1566$

(C) International Research Publication House. https://dx.doi.org/10.37624/IJERT/13.7.2020.1562-1566

[9] Bong-Young Kim, Zhixing Tian, Myung-Jin Bae. "A study on the causes of revenge psychology of Klaxon sound". International Journal of Engineering Research and Technology Volume 12, Issue 12, 2019, Pages 29472952.

[10] "Heart Rate Variability (HRV) features: can we use SDNN instead of rMSSD? A data-driven perspective on short term variability analysis". [https://www.hrv4training.com/blog/heart-ratevariability-hrv-features-can-we-use-sdnn-instead-ofrmssd-a-data-driven-perspective-on-short-termvariability-analysis]

[11] Bong-Young Kim, Myung-Jin Bae. "A study on the stress reduction effect of reading aloud the book using HRV". International Journal of Engineering Research and Technology Volume 12, Issue 9, 2019, Pages 1457-1461.
[12] S. Boonnithi and S. Phongsuphap, "Comparison of heart rate variability measures for mental stress detection," 2011 Computing in Cardiology, Hangzhou, 2011, pp. 8588.

[13] DAMIEN SABOUL1,2, VINCENT PIALOUX1, CHRISTOPHE HAUTIER. "The impact of breathing on HRV measurements: Implications for the longitudinal follow-up of athletes". European Journal of Sport Science, 2013 Vol. 13, No. 5, 534542.

[14] Bong-Young Kim, Myung-Jin Bae."A study on the effect of jogging on stress reduction". International Journal of Engineering Research and Technology 11(9). pp. 13631370.

[15] Pal G, Velkumary S, Madanmohan. "Effect of short-term practice of breathing exercises on autonomic functions in normal human volunteers ". Indian J Med Res 2004;120(2):115-21. 